\title{
S-Wave Borehole Tomography - A Tool for Geotechnical Site Characterization
}

\author{
Thomas Fechner \\ Geotomographie GmbH \\ Neuwied, Germany
}

\author{
Julius von Ketelhodt \\ Geotomographie GmbH \\ Neuwied, Germany
}

\author{
Zhiqing Lin \\ Earth Technologies Group,ETG \\ Guangzhou,China
}

\begin{abstract}
Geophysical methods are able to fill the gap between boreholes and to provide a measure of the spatial continuity of structures. Borehole seismic tomography promises the highest resolution when applied at a local scale of a few tens of meters. Currently, almost exclusively $\mathbf{P}$-wave tomography is employed in geotechnical oriented tomographic surveys. However, from the geotechnical perspective, the benefit of $P$ wave tomography is rather limited. It is the S-wave structure of the ground which is crucial to derive geotechnical relevant parameters, such as shear strength or other elastic moduli. Up to now, only little effort has been made to develop the borehole $S$ wave crosshole tomographic method. S-wave tomography has some clear advantages compared to conventional $P$-wave tomography, such as a better spatial resolution and a higher sensitivity to material changes. Furthermore, S-waves are only slightly influenced by the ground water table and S-waves passing this zone are not much affected by ray bending compared to P-waves. Within this paper we present first results of a newly developed S-wave tomography system and field results from different test sites.
\end{abstract}

Keywords—S-wave; borehole tomography; tool

\section{INTRODUCTION}

The soils shear modulus $G$ is an important parameter for foundation and construction design because it influences the force transfer between the structures and the soil. Depth dependent shear modulus profiles are needed as input to geotechnical numerical models. However, the shear modulus is strain dependent and in principle the dependency between shear modulus and shear strain needs to be described for a wide range of shear strains ${ }^{[1]}$ (Fig.1). Among these, starting values are needed, i.e. maximum shear modulus at small shear strain. The maximum shear modulus can be calculated from Swave measurements. Thus, S-wave velocity is directly linked to geotechnical problems.

Surface and borehole methods such as downhole and cross-hole testing are able to provide average S-wave velocity values. Among these methods cross-hole testing provides the most accurate and reliable values. However, the S-wave velocity is given as a function of depth only, with average values obtained from horizontal ray paths between a source and a receiver. Since the cross-hole testing method is applied between boreholes at small distances of $3-5 \mathrm{~m}$, it is not mean

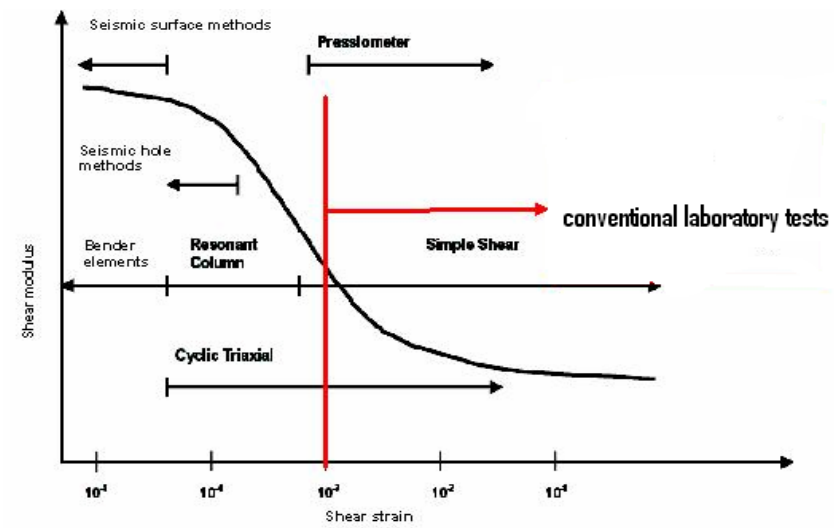

Fig. 1. Soil testing methods and typical strain ranges(after Mayne et al., 2000)

to resolve small scale structures between the boreholes. Consequently, the development of cross-hole S-wave tomography is required. Up to now, only little effort has been made to develop equipment enabling the competitive acquisition of S-wave cross-hole tomographic data. Possible reasons for this could be the availability of reliable borehole S-wave sources as well as borehole receivers with multiple seismic channels. Furthermore, the S-wave data acquisition is more time consuming compared to standard P-wave tomography and the subsequent processing of S-wave data requires more sophisticated and skilled personnel.

Within this paper we describe the technical solution for borehole S-wave tomography as well as the data processing and results.

\section{INSTRUMENTATION FOR BOREHOLE RECEIVERS}

Borehole geophones are routinely used for downhole applications. Usually, these are single receiver stations with only three geophone elements in a tri-axial arrangement. Coupling the borehole geophone is achieved by pneumatic clamping through an air bladder or through a mechanical arm which presses the geophone to the borehole wall. To perform S-wave tomography one needs several three component stations assembled to a single string to carry out the testing in an efficient way. The design of a multi-station borehole system needs to fulfil a few technical requirements, of which the most important are listed below. 


\section{3 geophones per station in a tri-axial arrangement}

Equal orientation of all horizontal stations required (all $\mathrm{X}-\mathrm{Y}$ components are aligned)

Light weight and easy handling

Robust coupling mechanism

8 or more stations per string to be efficient

In addition to technical requirements, also economical aspects were considered in the design of the system. A borehole system which allows an easy extension of seismic channels would be excellent as potential users could start with a few stations and can extend the string after their requirements. As typical seismic cables are good for 24 seismic channels they would be restricted to maximum of eight 3C stations. An extension would not be possible in this case.

To avoid limitation of the number of seismic channels a multi-station borehole receiver system was built with direct digitization at the geophones and digital data transmission. In case of using digital receivers there is no limitation of the number of $3 \mathrm{C}$ receivers. Further, a cable for digital data transmission is much lighter, smaller in diameter and less expensive compared to standard seismic cables. Thus, using digital components would allow a smart design of a multistation system by fulfilling the above mentioned general technical requirements.

The clamping mechanism of the borehole stations to the borehole wall is generally a critical technical issue. The clamping mechanism needs to be strong enough to hold the borehole geophone in position once it is in the borehole. The leading cable from surface needs to be tensionless to avoid mechanical interactions. It was decided to use a pneumatic clamping mechanism, in the form of pneumatic pressure cylinders. The advantage of using pneumatic pressure cylinders is that one can apply a high pressure rate regardless of the depth of the borehole geophones. Thus one could use bottled compressed air and a simple ON/OFF valve at a predefined pressure level to couple and de-couple the borehole geophones. In this case pneumatic clamping would be very easy and effective.

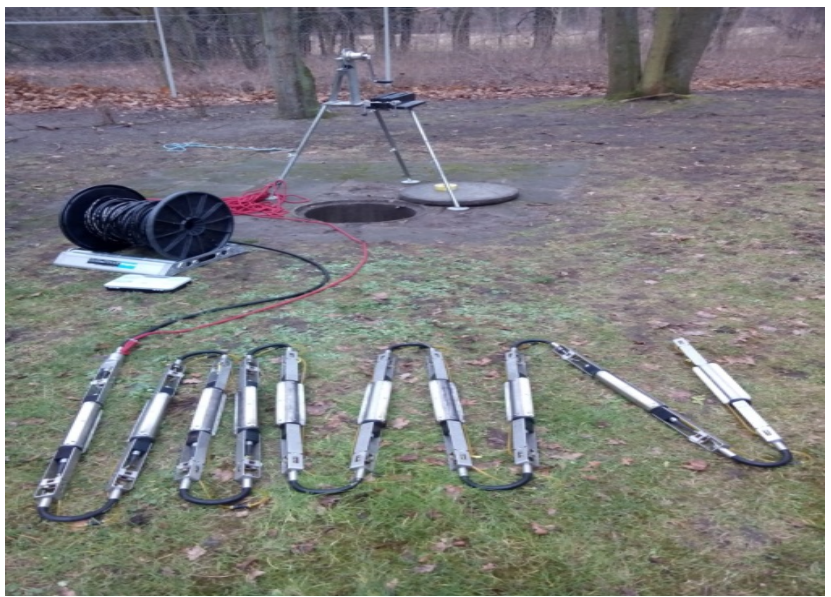

Fig. 2. Prototype Multi-station Borehole Acquisition System (MBAS)

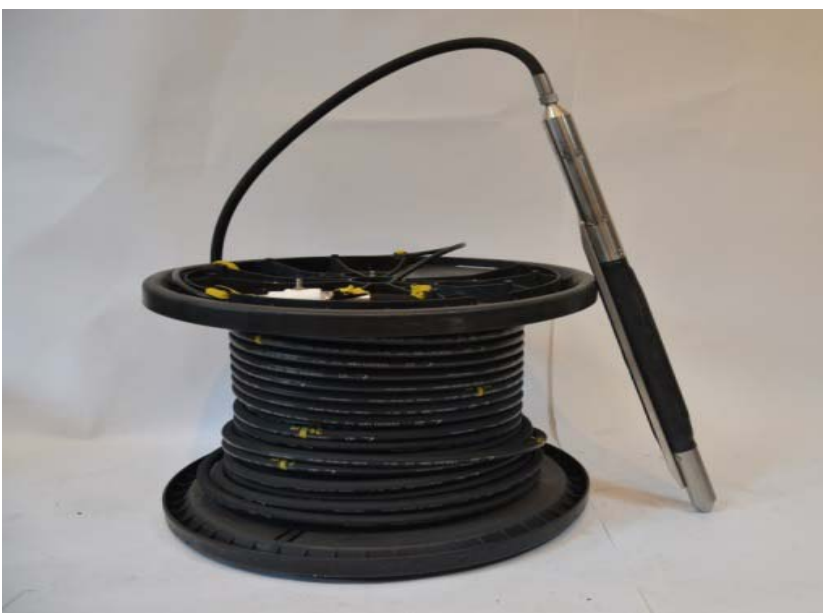

Fig. 3. S-wave source BIS-SH

The alignment of the different borehole stations in respect to each other is another issue that needs to be addressed. The proper identification of S-waves requires the sensors to be orientated in respect to the strike direction of the seismic source to receive the maximum wave amplitude. Usually, the orientation can be measured by using a magnetic compass. Alternatively, the orientation can also be controlled if the stations are connected to each other in the same direction and one can adjust the orientation of the whole string from surface by using a hydraulic hose. Hydraulic hoses can be coiled up on a drum but are inflexible and stiff against rotation. So, from surface the whole downhole array can be rotated to any desired direction. It was therefore decided to use a hydraulic hose to connect the single stations as well as to connect the stations to surface. Inside the hydraulic hose the digital transmission cable is placed as well as the air pressure supply. Thus, all cables are inside the hose which ensures good protection against cable damages.

As a prototype a multi-station borehole acquisition system (MBAS) was designed with 10 receiver stations (Fig.2).

\section{FIELD INSTRUMENTATION FOR BOREHOLE SOURCES}

To generate S-waves a borehole source type BIS-SH was used for field testing (Fig.3). This source produces mainly $\mathrm{SH}$ waves but also a significant amount of P-waves. The seismic signals are highly repeatable. The source works in dry or water filled boreholes and can be used in vertical or horizontal boreholes. Energy released by a high voltage supply discharges through a system of electromagnetic coils adjacent to a copper plate. The plate is then rejected causing the generation of a mechanical impact to the borehole wall. The borehole source is coupled to the borehole wall by a pneumatic clamping system (inflatable bladder) and the orientation of the source is controlled from surface by a torsional stiff hose.

\section{FIRST FIELD S-WAVE TOMOGRAPHY RESUlTS}

A field test was conducted at a site in Holland close to the German border. Three boreholes were available in an Lshaped arrangement, each borehole about 30m depth. The 
geology at the test site is mainly composed of unconsolidated sediments such as sand, gravel and clay. A P-wave tomography and an S-wave tomography survey was carried out, both with source and receiver intervals of $1 \mathrm{~m}$. A hydrophone string was used to receive P-waves and the MBAS was used to receive S-waves. The MBAS unit with seven 3C units at $1 \mathrm{~m}$ station interval was used. The MBAS unit was aligned with their X-sensor direction parallel to the source strike direction. Thus, these sensors should give the best seismic signal when evaluating the horizontally polarised shear wave.

Due to the good transmission properties of the soil, it was decided to use the same source for both the P- and S-wave tomographic measurements. Therefore, the SH-wave source type BIS-SH source was used for both $\mathrm{P}$ - and $\mathrm{S}$-wave measurements, as the BIS-SH generates both horizontally polarized shear waves (SH) and compressional waves $(\mathrm{P})$ with a high repeatability.

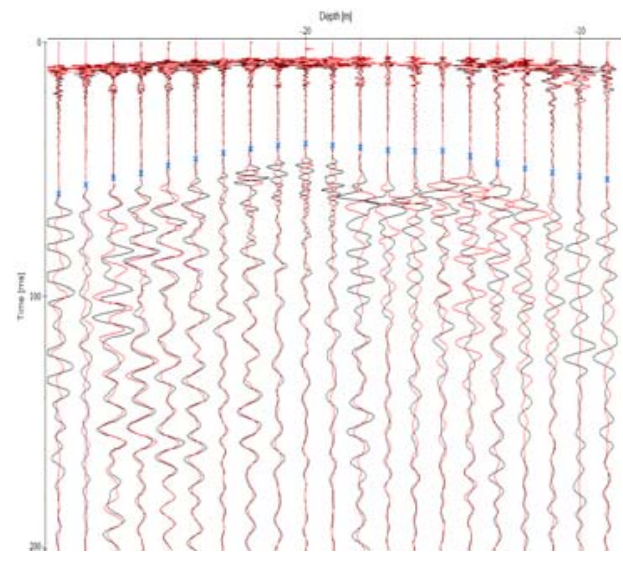

Fig. 4. : S-wave traveltime picks

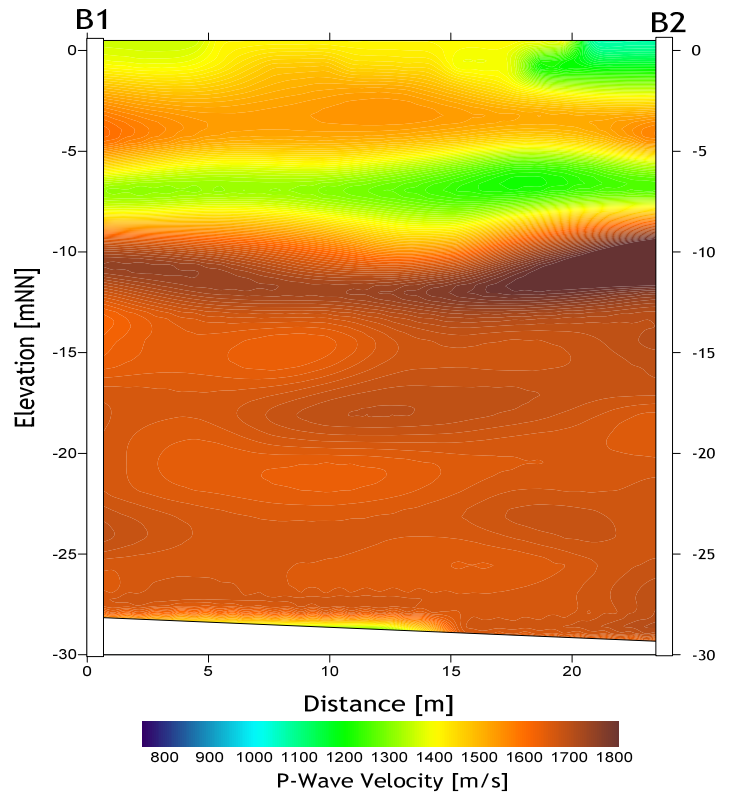

Fig. 5. Seismic P-wave tomogram

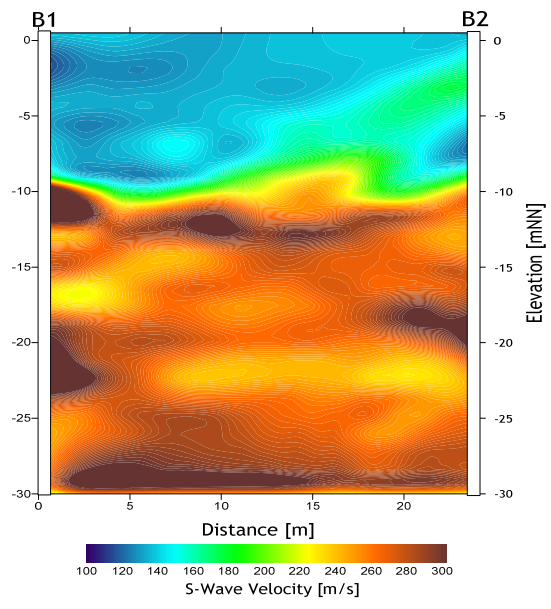

Fig. 6. Seismic S-wave tomogram

The seismic strike direction was aligned towards the receiver borehole in order to generate $\mathrm{P}$-waves with the highest amplitude. To generate S-waves, the seismic strike direction was aligned perpendicular to the receiver borehole. To get the opposite strike direction the source was rotated by $180^{\circ}$ and thus generating S-waves with opposing polarities. For each shot direction a separate seismic record was acquired and stored. Seismic traveltimes were picked for P- and Swaves. In total about 1800 traveltimes were determined for Pwave and about 950 traveltimes were analysed during the processing of the data for S-wave. Software GeoTomCG was used to carry out the SIRT traveltime inversion (Fig.4). Tomograms are shown in Fig.5 and 6.

\section{CONCLUSIONS}

A borehole seismic tomography experiment was successfully conducted using $\mathrm{P}$ - and S-wave. For S-wave tomography a newly developed digital multi-station acquisition borehole system was used to receive S-wave signals in an efficient way. A borehole source generating horizontally polarized S-wave was used. Seismic P- and Swave traveltimes were picked and inverted using the SIRT procedure. In general, the $\mathrm{P}$-wave and S-wave tomograms show the same geological units. However, more detailed structures are visible in the S-wave tomogram within the main aquifer zone below $10 \mathrm{~m}$. This seems to be mainly due to the higher dynamic range of the S-wave which is about 1.5 for Swave and only 1.2 for $\mathrm{P}$-wave. Average wavelength of the seismic wave is in the range of $1 \mathrm{~m}$ for both $\mathrm{P}$ - and S-wave indicating that both wave types have the same structural resolution. Considering the results, S-wave tomography seems to be a very promising and complementary tool to P-wave tomography surveys where higher sensitivity is required and soil dynamic parameters are needed.

\section{REFERENCES}

[1] Mayne, P.W., Schneider, J.A., Martin, G.K. (2000): Small- and large strain soil properties from seismis flat dilatometer test, in: Pre-failure Deformation haracteristics of geomaterials, Balkema, M. Jamiolkowski; R. Lancelotta: LoPresti (eds). 